\title{
Risk Factors of Postoperative Clinically Relevant Pancreatic Fistula following Distal Pancreatectomy with Stapler Closure
}

\author{
Sefa Ozyazici and Osman Erdogan ${ }^{2}$ \\ ${ }^{1}$ Department of General Surgery, Adana City Training and Research Hospital, Adana, Turkey \\ ${ }^{2}$ Department of Surgical Oncology, Adana City Training and Research Hospital, Adana, Turkey
}

\begin{abstract}
Objective: To analyse clinicopathological variables to determine risk factors of postoperative pancreatic fistula in patients having distal pancreatectomy with stapler closure.

Study Design: Observational study.

Place and Duration of Study: Adana City Training and Research Hospital, Adana, Turkey, from January 2015 to May 2020.

Methodology: Medical files of 38 cases having distal pancreatectomy with stapler closure of the pancreatic remnant for conditions emerging from the pancreas or other organs were analysed. Data about demographics, perioperative and pathological findings, postoperative outcomes were gathered. Univariate analysis was utilised for comparisons of categorical data. The possible risk factors found to be significant in the univariate analysis were included into the logistic regression analysis.

Results: Based on the description reported by the International Study Group of Pancreatic Surgery, 23.7\% and 31.6\% of the patients had biochemical leakage and clinically relevant postoperative pancreatic fistula (POPF) respectively. Univariate and multivariate analyses made to determine risk factors of POPF showed a relation between stapler closure alone $(p=0.018)$ and soft parenchymal texture $(p=0.002)$ and clinically relevant POPF. Multivariate analyses revealed that parenchymal texture was the sole independent predictor of clinically relevant POPF (OR, 0.016, $p=0.039)$.

Conclusion: Soft pancreatic texture is independently predictive of clinically relevant POPF following distal pancreatectomy with stapler closure. However, reinforcement of the stapler line with extra sutures decreases the risk of clinically relevant POPF.
\end{abstract}

Key Words: Distal pancreatectomy, Pancreatic fistula, Stapler closure, Suture reinforcement.

How to cite this article: Ozyazici S, Erdogan O. Risk Factors of Postoperative Clinically Relevant Pancreatic Fistula following Distal Pancreatectomy with Stapler Closure. J Coll Physicians Surg Pak 2021; 31(10):1214-1218.

\section{INTRODUCTION}

Distal pancreatectomy is commonly utilised for the treatment of various malignant and non-malignant conditions affecting the pancreas except for its head. It has recently been reported to be indicated for the treatment of pancreatic lesions, non-pancreatic malignancies, chronic pancreatitis and trauma. At present, the mortality following distal pancreatectomy has decreased to $0-7 \% .{ }^{1}$ On the other hand, in spite of developments in the operative procedure and perioperative patient care, the morbidity rate remains to be high, varying between $37 \%$ and $50 \%$ in the past ten years. ${ }^{2}$

Correspondence to: Dr. Osman Erdogan, Department of Surgical Oncology, Adana City Training and Research Hospital, Adana, Turkey

E-mail: osman erdogan85@hotmail.com

Received: January 05, 2021; Revised: February 10, 2021; Accepted: March 08, 2021

DOI: https://doi.org/10.29271/jcpsp.2021.10.1214
Postoperative pancreatic fistula (POPF) is the most frequent complication emerging after distal pancreatectomy. Postsurgical morbidity is linked with many more problems, like intra-abdominal abscess, sepsis, and hemorrhage. ${ }^{3}$ Among other features creating risk are the structure of the pancreas, the width of the main pancreatic duct, method of resection and way of closing the pancreatic remnant and experience of the surgeon. Despite the fact that numerous methods have been reported to eliminate POPF, there is no agreement on avoidance of POPF. While some studies have revealed that stapler closure and hand-sewn closure are not different at all in terms of POPF, other studies have shown that stapler closure is reliable. ${ }^{4,5} \mathrm{At}$ the study place, stump closure was performed using the stapling technique.

The International Study Group of Pancreatic Fistula (ISGPF) reported an agreement about categorisation of POPF and improved the description made by the International Study Group of Pancreatic Surgery (ISGPS) in 2016. ${ }^{6,7}$ Based on ISGPS criteria, biochemical leakage indicates grade A POPF, while clinically relevant POPF is indicator of grades $B$ and $C .{ }^{8}$ It is stated in 
the literature that clinically relevant POPF appears in 14.8-27.7\% of the cases and is the most complex complication, secondary to distal pancreatectomy. ${ }^{8}$ For this reason, it is crucial for surgeons to reveal risk factors of clinically relevant POPF.

The objective of the current study was to determine risk factors of clinically relevant POPF following distal pancreatectomy using staplerclosure.

\section{METHODOLOGY}

Medical files of 38 cases having distal pancreatectomy with stapler closure of the pancreatic remnant for conditions emerging from the pancreas or other organs at Adana City Training and Research Hospital, Adana, Turkey, from January 2015 to May 2020 were analysed. Data about demographics like age, gender, body mass index (BMI), perioperative and pathological findings, postoperative outcomes, conditions requiring surgical treatment, ASA status, pancreatic texture, estimated blood loss (EBL), duration of surgery, surgical technique, pancreatic fistula and postoperative outcomes including complications and death were gathered. The study received approval from the Ethical Review Committee (ERC) of the Institution (61/988/08.07.2020).

Distal pancreatectomy was carried out either as the primary operation or as a component of surgery for multiorgan removal to treat a malignancy outside the pancreas. The surgery performed included distal pancreatectomy protecting the spleen, distal pancreatectomy accompanied by splenectomy, or distal pancreatectomy combined with splenectomy and removal of the neighboring organ. Neighboring organ removal referred to removal of minimum one more organ along with the distal pancreas and spleen. Pancreatic parenchymal closure techniques involved stapled closure alone or stapled closure with suture reinforcement.

The description of POPF was based on the report by the ISGPS. ${ }^{9}$ POPF was categorised into absence of fistula, biochemical signs of fistula including surgical drain amylase three times higher compared to its serum levels with no clinical results (biochemical leakage), biochemical signs of fistula treated with constant drainage over three weeks, endoscopic or percutaneous drainage, angiography for hemorrhage, or symptoms of infection not accompanied by organ insufficiency (grade B), biochemical signs of fistula requiring reoperation or causing organ insufficiency or death (grade C). In the present research, grades $B$ and $C$ were considered as clinically relevant POPF.

Pancreatic stiffness was defined as either hard or soft by the surgeon performing the operation at the time of transection. The degree of surgical morbidity was determined, based on the Clavien-Dindo classification. ${ }^{9}$ Complications of grade $\geq$ III were regarded as major. Any death in hospital or in 30 days after distal pancreatectomy was accepted as the 30-day mortality. All patients having $\mathrm{BMI} \geq 25 \mathrm{Kg} / \mathrm{m}^{2}$ were considered as overweight, based on the description specified by the World Health Organisation (WHO).
All surgeries were carried out by the same surgical team. Resection was made by using conventional surgery. Stump closure of the remnant pancreas was mainly performed by utilising two techniques, i.e. stapled closure alone or stapled closure with suture reinforcement. A stapling device with blue or green cartridges was used across the whole pancreas body depending on the thickness of the pancreas (linear stapler, Ethicon linear cutter Sr 75 mm US, LLC) and «U» sutures passing through each other with 2-0 polypropylene suture of the whole stump were put. An abdominal drain was also inserted over the pancreas stump.

Data analysis was made with the statistical package programme for social sciences version 25.0 (SPSS Inc. Chicago, IL, USA). Results of descriptive statistics were presented by using mean $\pm S D$, frequencies and percentages. The Chi-square or Fisher's Exact test for univariate analysis was utilised for comparisons of categorical data between the patients. Logistic regression test was carried out on the parameters having $p$ $<0.25$ in the univariate statistical analysis as possible conditions creating risk. The odds ratio (OR) with a 95\% confidence interval was determined. $P<0.05$ was accepted as significant.

Table l: Patient demographics $(\mathbf{n}=\mathbf{3 8})$.

\begin{tabular}{|l|l|}
\hline Age (years) & $52.4 \pm 17.9$ \\
\hline Sex & $16(42.1 \%)$ \\
\hline Male & $22(57.9 \%)$ \\
\hline Female & $26.9 \pm 5.2$ \\
\hline Body mass index (kg/m2) & $3.69 \pm 0.49$ \\
\hline Preoperative serum albumin (g/dL) \\
\hline Disease: Indications for surgery \\
\hline Pancreatic & $32(84.2 \%)$ \\
\hline Cystic neoplasm of the pancreas & $9(23.7 \%)$ \\
\hline Pancreatic adenocarcinoma & $8(21.1 \%)$ \\
\hline Pancreatic neuroendocrine tumor & $6(15.8 \%)$ \\
\hline Solid pseudopapillary tumor & $5(13.2 \%)$ \\
\hline Chronic pancreatitis & $2(5.3 \%)$ \\
\hline Impacted stent & $1(2.6 \%)$ \\
\hline Pancreatic pseudocyst & $1(2.6 \%)$ \\
\hline Non-pancreatic & $6(15.8 \%)$ \\
\hline Gastric adenocarcinoma & $3(7.9 \%)$ \\
\hline Colonic adenocarcinoma & $2(5.3 \%)$ \\
\hline Spleen mass & $1(2.6 \%)$ \\
\hline ASA level ( $n=38)$ & $6(15.8 \%)$ \\
\hline 1 & $14(36.8 \%)$ \\
\hline 2 & $17(44.7 \%)$ \\
\hline 3 & $1(2.6 \%)$ \\
\hline 4 &
\end{tabular}

\section{RESULTS}

Demographics and surgical indications of the patients are shown in Table I. Out of 38 patients undergoing distal pancreatectomy, 16 (42.1\%) were males and 22 (57.9\%) were females. The patients were aged 21-82 years with a mean of $52.4 \pm 17.9$ years, and the mean BMI was $26.9 \pm 5.2 \mathrm{Kg} / \mathrm{m}^{2}$. The mean albumin level was $3.69( \pm 0.49)$. The majority of the patients were at ASA III status; and $44.7 \%$ of the patients had at least one additional disease. Concerning indications for surgery, 32 patients $(84.2 \%)$ had pancreatic disease and six patients (15.8\%) had non-pancreatic disease. The most common 
pancreas-related indication was cystic neoplasms and the most common non-pancreatic cause was gastric cancer.

Table II: Operative features and postoperative outcomes.

\begin{tabular}{|l|l|}
\hline Operative time & $229.7 \pm 38.8$ \\
\hline Intraoperative blood loss $(\mathrm{mL})$ & $255.5 \pm 106.6$ \\
\hline Pancreatic texture $(n=38)$ & $21(55.3 \%)$ \\
\hline Soft & $17(44.7 \%)$ \\
\hline Firm & $22(57.9 \%)$ \\
\hline Type of surgery $(n=38)$ & $9(23.7 \%)$ \\
\hline Distal pancreatectomy with Splenectomy & $7(18.4 \%)$ \\
\hline $\begin{array}{l}\text { Distal pancreatectomy with splenectomy with } \\
\text { additional organ resection }\end{array}$ & $21(55.3 \%)$ \\
\hline Spleen-preserving distal pancreatectomy & $17(44.7 \%)$ \\
\hline Closure type $(n=38)$ & $1(2.6 \%)$ \\
\hline Stapler alone & $25(65.8 \%)$ \\
\hline Stapler with suture reinforcement & $9(23.7 \%)$ \\
\hline Death & $12(31.6 \%)$ \\
\hline Complications & $9(23.7 \%)$ \\
\hline Biochemical leak & $3(7.9 \%)$ \\
\hline Pancreatic fistula & \\
\hline Grade B &
\end{tabular}

Table III: Univariate analysis of potential clinicopathological and operative variables associated with clinically relevant POPF after distal pancreatectomy.

\begin{tabular}{|c|c|c|c|}
\hline & \multicolumn{2}{|c|}{ POPF ISGPF 2016} & \multirow[b]{2}{*}{$\mathbf{p}$} \\
\hline & Yes & No & \\
\hline \multicolumn{4}{|l|}{ Age (years) } \\
\hline$<60$ & $8(38.1 \%)$ & $13(61.9 \%)$ & 0.337 \\
\hline$\geq 60$ & $4(23.5 \%)$ & $13(76.5 \%)$ & \\
\hline \multicolumn{4}{|l|}{ Sex } \\
\hline Male & $4(25 \%)$ & $12(75 \%)$ & 0.457 \\
\hline Female & $8(36.4 \%)$ & $14(63.6 \%)$ & \\
\hline \multicolumn{4}{|l|}{ ASA } \\
\hline ASA 1-2 & $7(35 \%)$ & $13(65 \%)$ & 0.632 \\
\hline ASA 3-4 & $5(27.8 \%)$ & $13(72.2 \%)$ & \\
\hline \multicolumn{4}{|l|}{ BMI $\left(\mathrm{kg} / \mathrm{m}^{2}\right)$} \\
\hline Normal $(<25)$ & $3(20 \%)$ & $12(80 \%)$ & 0.294 \\
\hline Overweight - obese ( $\geq 25$ ) & $9(39.1 \%)$ & $14(60.9 \%)$ & \\
\hline \multicolumn{4}{|l|}{ Operative time (min) } \\
\hline$<240$ & $8(38.1 \%)$ & $13(61.9 \%)$ & 0.337 \\
\hline$\geq 240$ & $4(23.5 \%)$ & $13(76.5 \%)$ & \\
\hline \multicolumn{4}{|l|}{ Blood loss (mL) } \\
\hline$<300$ & $7(30.4 \%)$ & $16(69.6 \%)$ & $>0.999$ \\
\hline$\geq 300$ & $5(33.3 \%)$ & $10(66.7 \%)$ & \\
\hline \multicolumn{4}{|l|}{ Texture of pancreatic parenchyma } \\
\hline Soft & $11(52.4 \%)$ & $10(47.6 \%)$ & 0.002 \\
\hline Hard & $1(5.9 \%)$ & $16(94.1)$ & \\
\hline \multicolumn{4}{|l|}{ Additional organ resection } \\
\hline Yes & $3(27.3 \%)$ & $8(72.7 \%)$ & $>0.999$ \\
\hline No & $9(33.3 \%)$ & $18(66.7 \%)$ & \\
\hline \multicolumn{4}{|l|}{ Spleen preserving } \\
\hline Yes & $4(57.1 \%)$ & $3(42.9 \%)$ & 0.176 \\
\hline No & $8(25.8 \%)$ & $23(74.2 \%)$ & \\
\hline \multicolumn{4}{|l|}{ Closure type } \\
\hline Stapler alone & $10(47.6 \%)$ & $11(52.4 \%)$ & 0.018 \\
\hline Stapler with suture reinforcement & $2(11.8 \%)$ & $15(88.2 \%)$ & \\
\hline
\end{tabular}

Operative information and technical details are outlined in Table II. The mean operation time was 229.7 minutes ( \pm 38.8 ) and EBL ranged between $100 \mathrm{ml}$ and $550 \mathrm{ml}(255.5 \pm 106.6 \mathrm{ml})$. Twenty-one patients $(55.3 \%)$ had soft pancreatic tissue. Twenty-two patients $(57.9 \%)$ had distal pancreatectomy with splenectomy, and nine patients $(23.7 \%)$ had distal pancreatectomy with splenectomy, and one or more additional procedures. Seven patients (18.4\%) had spleen preserving distal pancreatectomy; they all had benign or borderline diseases. The most frequently resected non-pancreatic organ (except for the spleen) was the stomach $(n=6)$. Among the other resected organs were colon $(n=2)$ and gallbladder $(n=1)$. Pancreatic parenchyma was closed by stapling alone in 21 patients (55.3\%) and stapler with suture reinforcement in 17 patients (44.7\%).

Postsurgical problems and mortality are summarised in Table II. The mortality and complication rates in the postoperative period were $2.6 \%$ and $65.8 \%$ respectively. In the postoperative period, death occurred in one patient (2.6\%). The patient had a pancreatic and esophago-jejunal fistula after 39 years of gastric cancer due to total gastrectomy, splenectomy and distal pancreatectomy. He died due to sepsis after reoperation. Grade 3 had a higher complication rate of $28.9 \%(n=11)$. The most common complication was pancreatic fistula. In fact, 12 patients (31.6\%) developed pancreatic fistula. Out of 12 patients, 9 (75\%) were classified as grade B and 3 (25\%) were classified as grade C. Nine patients (23.7\%) had biochemical leaks. Death occurred in one of the patients with pancreatic leakage.

Two patients (5.3\%) needed reoperation, one of whom had both a pancreatic fistula and esophagojejunal fistula. He died of sepsis after surgery. The other patient was reoperated due to intra-abdominal abscess, secondary to type C pancreatic fistula. She wastreated with percutaneousdrainage in theintensive care unit due to intraabdominal abscess and renal failure; and was discharged on the 12th day after surgery.

Univariate analysis was made to analyse the relation between any risk factors and pancreatic fistula development (Table III). None of the variables such as age, gender, ASA status, BMI, duration of operation, $\mathrm{EBL}$, neighbouring organ resection and spleen preserving, had a significant relation with emergence of clinically relevantpancreatic fistula. In the univariateanalysis, softpancreatic texture $(p=0.002)$ and stapler closure alone $(p=0.018)$ had a significant relationship with clinically relevant pancreatic fistula. The only independent risk factor for clinically relevant pancreatic fistula was soft parenchymal texture in multivariate analysis ( $p=0.039$; OR: $0.016 \% 95 \mathrm{Cl} 0.000-0.810$ ).

\section{DISCUSSION}

Risk factors for POPF following distal pancreatectomy remain uncertain and have been previously reported using severaltechniques. ${ }^{10}$ Indeed, gender, age, stapler closure, BMI, diabetes mellitus, soft pancreatic texture, pancreatic thickness, chronic pancreatitis, extended lymphadenectomy, hand suture closure, additional organ resection, failure of main pancreatic duct (MPD) ligation, and operation time were described as risk factors. ${ }^{3,4}$ The risk factors for POPF following distal pancreatectomy utilising staples have not yet been clarified. Therefore, this study was directed towards determining them.

A systematic review by Cecka et al. using different criteria showed that POPF rates in distal pancreatectomy ranged from $3.7 \%$ to $68.5 \% .{ }^{11}$ The clinically relevant POPF (Grade B, C) rate using ISGPS in this study was $31.6 \%$. It is not significantly 
different from the ones revealed by the DISPACT study (stapler $32 \%$ ) and other studies. ${ }^{5-7}$

In terms of surgery, POPF still remains to be solved. It has been pointed out in the literature that the closure procedure of the remnant pancreatic stump is primarily responsible for POPF development. ${ }^{11-13}$ For this reason, various stump closure techniques closure techniques have been reported and there is an agreement about the technique responsible for POPF. ${ }^{12-14}$ Univariate analysis showed a significant rise in POPF development when the remaining pancreatic remnant was closed with only staples. In 2010, Heng etal. reported that closure of pancreatic stump with stapler alone was an independent predictor of POPF. ${ }^{14}$ Yoshioka et al. demonstrated that $50 \%$ of their cases had POPF when closure of all pancreatic stumps was performed by utilising stapler alone. ${ }^{15}$ In the DISPACT study in 2011, suture closure was compared with staple closing techniques, and the latter was found to create a higher rate of POPF $(36 \%) .{ }^{5}$ In the current study, the POPF ratio was $47.6 \%$ (10/21) in the cases in whom closure of the remnant pancreatic stump was carried out by employing stapler alone. Based on the abovementioned evidence, it can be suggested that closing the pancreatic remnant stump solely with stapler is unsecure.

In the current study, the POPF rate was $11.8 \%(2 / 17)$ when stapler and sutures were utilised in combination to close the remnant pancreatic stump. Likewise, a study with a large number of patients in 2009 showed a reduction in the POPF rate (6\%) following the use of staples and sutures together ${ }^{16}$. Yuksel and Bostanci et al. reported that utilization of additional sutures in the stapler line decreases the risk of POPF. ${ }^{17}$ Ecker etal. found that the POPF level was lower in those who had suture reinforcement in the staple line compared to those that were closed with only staples in a multicentre study. ${ }^{12}$ The findings mentioned above show that closing the remaining pancreatic root with staples and sutures is a safer technique.

The current study showed that the soft pancreatic texture is an independent risk factor for clinically relevant POPF when a linear stapler was utilised (OR: 0.016, $p=0.039$ ). It has been stated in the literature that soft pancreas is an important risk factor of POPF as well. ${ }^{18,19}$ Unek et al. found in their study in 2013 that soft texture of the pancreatic parenchyma created risk for POPF (OR: 12.420, p-value $=0.048$ ) and that POPF could be decreased by using "U" shaped sutures. ${ }^{19}$ Mendoza et al. reported that pancreatic parenchyma on its own does not independently predict POPF, but that thickness of the pancreas is an important risk factor of POPF. ${ }^{20}$ In addition, they pointed out that both thickness and softness together play an important role in POPF. When staple closure was utilised, a thicker pancreatic stump produces a rise in POPF following distal pancreatectomy. ${ }^{21,22}$ It seems that staple closure is suitable at least for the thin pancreas. ${ }^{23}$ In the present study, data about thickness of the pancreas and staple length could not be accessed. Therefore, the relation between stapler, pancreatic tissue, and thickness could not be evaluated. In light of the relevant literature, POPF is likely to be mainly related to pancreatic tissue, thickness and closure technique. More clinical trials are needed on the appropriateness of the closure technique for pancreatic tissue and thickness.

In a study with 232 consecutive patients undergoing distal pancreatectomy, Goh et al. found a mortality rate of $3 \%$ and a morbidity rate of $47 \%$ by using the Dindo et al.'s classification. ${ }^{13}$ Kleef et al. reported a mortality rate of $2 \%$ in 302 consecutive patients who underwent distal pancreatectomy. ${ }^{24}$ In the current study, the mortality rate of the patients was $2.6 \%$, the morbidity rate was $65.8 \%$, the major complication rate of grade 3 and higher grades was $28.9 \%$. Therefore, the morbidity and mortality rates found in this study were similar to those revealed in studies using similar criteria to identify complications.

This study has two limitations. The study has a retrospective design and data about the width of the main pancreatic duct, the thickness of the pancreatic stump, and the staple length could not be gathered, which is likely to affect the results. In addition, postoperative results could have been collected and evaluated in more detail.

\section{CONCLUSION}

Pancreatic fistula is still the most frequent complication, secondary to distal pancreatectomy. Soft pancreatic parenchyma was an independent predictor of clinically relevant POPF. Distal pancreatectomy can belinked with a greater predisposition to clinically relevant POPF if the pancreatic remnant is closed only with staplers. However, the risk of POPF decreases when additional sutures are utilised.

\section{CONFLICT OF INTEREST:}

The authors declared no conflict of interest.

\section{PATIENTS' CONSENT:}

Consents for publication were obtained from patients whose data are included in this manuscript.

\section{AUTHORS' CONTRIBUTION:}

SO, OE: Collected data, written manuscript, edited and critically reviewed the manuscript.

\section{REFERENCES}

1. Kawai M, Hirono S, Okada K, Sho M, Nakajima Y, Eguchi H, et al. Randomised controlled trial of pancreaticojejunostomy versus stapler closure of the pancreatic stump during distal pancreatectomy to reduce pancreatic fistula. Ann Surg 2016; 264(1):180-7. doi: 10.1097/SLA.00000 00000001395.

2. Okano K, Hirao T, Unno M, Fujii T, Yoshitomi H, Suzuki S, et al. Postoperative infectious complications after pancreatic resection. BrJ Surg 2015; 102(12):1551-60. doi: 10.1002/ bjs.9919.

3. Peng YP, Zhu XL, Yin LD, Zhu Y, Wei JS, Wu JL, et al. Risk factors of postoperative pancreatic fistula in patients after distal pancreatectomy: A systematic review and metaanalysis. Sci Rep 2017; 7(1):185. doi: 10.1038/s41598017-00311-8. 
4. Zhang $\mathrm{H}$, Zhu F, Shen M, Tian R, Shi CJ, Wang X, et al. Systematic review and meta-analysis comparing three techniques for pancreatic remnant closure following distal pancreatectomy. Br J Surg 2015; 102(1):4-15. doi: $10.1002 /$ bjs. 9653

5. Diener MK, Seiler CM, Rossion I, Kleeff J, Glanemann M, Butturini $G$, et al. Efficacy of stapler versus hand-sewn closure after distal pancreatectomy (DISPACT): A randomised, controlled multicentre trial. Lancet (London, England) 2011; 377(9776):1514-22. doi: 10.1016/S01406736(11)60237.

6. Bassi C, Marchegiani G, Dervenis C, Sarr M, Abu Hilal M, Adham $M$, et al. International study group on pancreatic surgery (ISGPS). The 2016 update of the International study group (ISGPS) definition and grading of postoperative pancreatic fistula: 11 Years After. Surgery 2017; 161(3): 584-91. doi: 10.1016/j.surg.2016.11.014.

7. Yang F, Jin C, Hao S, Fu D. Drain contamination after distal pancreatectomy: Incidence, risk factors, and association with postoperative pancreatic fistula. J Gastrointest Surg 2019; 23(12):2449-58. doi: 10.1007/s11605-019-04155-7.

8. Van Buren G 2nd, Bloomston M, Schmidt CR, Behrman SW, Zyromski NJ, Ball CG, et al. A Prospective randomised multicenter trial of distal pancreatectomy with and without routine intraperitoneal drainage. Ann Surg 2017; 266(3): 421-31. doi: 10.1097/SLA.0000000000002375.

9. Dindo D, Demartines $N$, Clavien PA. Classification of surgical complications: A new proposal with evaluation in a cohort of 6336 patients and results of a survey. Ann Surg 2004; 240(2): 205-13. doi: 10.1097/01.sla.000013 3083.54934.ae.

10. Kollár D, Huszár T, Pohárnok Z, Cselovszky É, Oláh A. A Review of techniques for closure of the pancreatic remnant following distal pancreatectomy. Dig Surg 2016; 33(4): 320-8. doi: 10.1159/000445017.

11. Cecka F, Jon B, Subrt Z, Ferko A. Surgical technique in distal pancreatectomy: A systematic review of randomised trials. BioMed Res Intern 2014; 2014:482906. doi: 10.1155/ 2014/482906

12. Ecker BL, McMillan MT, Allegrini V, Bassi C, Beane JD, Beckman RM, et al. Risk factors and mitigation strategies for pancreatic fistula after distal pancreatectomy: Analysis of 2026 resections from the International, multi-institutional distal pancreatectomy study group. Ann Surg 2019; 269(1):143-149. doi: 10.1097/SLA.0000000000002491.

13. Goh BK, Tan YM, Chung YF, Cheow PC, Ong HS, Chan WH, et al. Critical appraisal of 232 consecutive distal pancreatectomies with emphasis on risk factors, outcome, and management of the postoperative pancreatic fistula: A 21-year experience at a single institution. Arch Surg 2008; 143(10): 956-65. doi: 10.1001/archsurg.143.10.956.
14. Kah Heng CA, Salleh I, San TS, Ying F, Su-Ming T. Pancreatic fistula after distal pancreatectomy: Incidence, risk factors and management. ANZ J Surg 2010; 80: 619-23. doi: 10.1111/j.1445-2197.2010.05337.x.

15. Yoshioka R, Saiura A, Koga R, Seki M, Kishi Y, Morimura R, et al. Risk factors for clinical pancreatic fistula after distal pancreatectomy: Analysis of consecutive 100 patients. World J Surg 2010; 34(1):121-5. doi: 10.1007/s00268009-0300-3.

16. Nathan H, Cameron JL, Goodwin CR, Seth AK, Edil BH, Wolfgang $\mathrm{CL}$, et al. Risk factors for pancreatic leak after distal pancreatectomy. Ann Surg 2009; 250(2):277-81 doi: 10.1097/SLA.0b013e3181ae34be.

17. Yüksel A, Bostancı EB, Çolakoğlu MK, Ulaş M, Özer i, Karaman $\mathrm{K}$, et al. Pancreatic stump closure using only stapler is associated with high postoperative fistula rate after minimal invasive surgery. Turk J Gastroenterol 2018; 29(2):XXXX. doi: 10.5152/tjg.2018.17567

18. Okano K, Kakinoki K, Suto $H$, Oshima M, Kashiwagi $H$, Yamamoto $\mathrm{N}$, et al. Persisting ratio of total amylase output in drain fluid can predict postoperative clinical pancreatic fistula. J Hepato Biliarypancreatic Sci 2011; 18(6):815-20. doi: 10.1007/s00534-011-0393-6.

19. Unek T, Egeli T, Ozbilgin M, Arslan NC, Astarcioglu H, Karademir $\mathrm{S}$, et al. Retrospective analysis of risk factors affecting pacreatic fistula formation after the closure of the pancreatic stump with sutures in distal pancreatectomy. Hepato-gastroenterology 2013; 60(127):1778-84.

20. Mendoza AS 3rd, Han HS, Ahn S, Yoon YS, Cho JY, Choi Y. Predictive factors associated with postoperative pancreatic fistula after laparoscopic distal pancreatectomy: A 10-year single-institution experience. Surgical Endoscopy 2016; 30(2):649-56. doi: 10.1007/s00464-015-4255-1.

21. Kawai M, Tani M, Okada K, Hirono S, Miyazawa M, Shimizu $A$, et al. Stump closure of a thick pancreas using stapler closure increases pancreatic fistula after distal pancreatectomy. Am J Surg 2013; 206(3):352-9. doi: 10.1016/j. amjsurg.2012.11.02.

22. Kim H, Jang JY, Son D, Lee $S$, Han $Y$, Shin YC, et al. Optimal stapler cartridge selection according to the thickness of the pancreas in distal pancreatectomy. Medicine (Baltimore) 2016; 95(35):e4441. doi:10.1097/MD.0000000000004441.

23. Eguchi $H$, Nagano $H$, Tanemura M, Takeda $Y$, Marubashi S, Kobayashi S, et al. A thick pancreas is a risk factor for pancreatic fistula after a distal pancreatectomy: selection of the closure technique according to the thickness. Dig Surg 2011; 28(1):50-6. doi: 10.1159/000322406.

24. Kleeff J, Diener MK, Z'graggen K, Hinz U, Wagner M, Bachmann J, et al. Distal pancreatectomy: Risk factors for surgical failure in 302 consecutive cases. Ann Surg 2007; 245(4):573-82. doi: 10.1097/01.sla.0000251438.43135.fb. 